\title{
Forward Stochastic Reachability Analysis for Uncontrolled Linear Systems using Fourier Transforms
}

\author{
Abraham P. Vinod \\ Electrical \& Comp. Eng. \\ University of New Mexico \\ Albuquerque, NM 87131, USA \\ aby.vinod@gmail.com
}

\author{
Baisravan \\ HomChaudhuri \\ Electrical \& Comp. Eng. \\ University of New Mexico \\ Albuquerque, NM 87131, USA \\ baisravan.hc@gmail.com
}

\author{
Meeko M. K. Oishi \\ Electrical \& Comp. Eng. \\ University of New Mexico \\ Albuquerque, NM 87131, USA \\ oishi@unm.edu
}

\begin{abstract}
We propose a scalable method for forward stochastic reachability analysis for uncontrolled linear systems with affine disturbance. Our method uses Fourier transforms to efficiently compute the forward stochastic reach probability measure (density) and the forward stochastic reach set. This method is applicable to systems with bounded or unbounded disturbance sets. We also examine the convexity properties of the forward stochastic reach set and its probability density. Motivated by the problem of a robot attempting to capture a stochastically moving, non-adversarial target, we demonstrate our method on two simple examples. Where traditional approaches provide approximations, our method provides exact analytical expressions for the densities and probability of capture.
\end{abstract}

\section{CCS Concepts}

-Theory of computation $\rightarrow$ Stochastic control and optimization; Convex optimization; •Computing methodologies $\rightarrow$ Control methods; Computational control theory;

\section{Keywords}

Stochastic reachability; Fourier transform; Convex optimization

\section{INTRODUCTION}

Reachability analysis of discrete-time dynamical systems with stochastic disturbance input is an established tool to provide probabilistic assurances of safety or performance and has been applied in several domains, including motion planning in robotics 1, 2, spacecraft docking [3], fishery management and mathematical finance 4], and autonomous survelliance 5]. The computation of stochastic reachable and viable sets has been formulated within a dynamic programming framework [4, 6] that generalizes to discrete-time stochastic hybrid systems, and suffers from the well-known

Permission to make digital or hard copies of all or part of this work for personal or classroom use is granted without fee provided that copies are not made or distributed for profit or commercial advantage and that copies bear this notice and the full citation on the first page. Copyrights for components of this work owned by others than ACM must be honored. Abstracting with credit is permitted. To copy otherwise, or republish, to post on servers or to redistribute to lists, requires prior specific permission and/or a fee. Request permissions from permissions@ acm.org.

HSCC '17, April 18-20, 2017, Pittsburgh, PA, USA

(c) 2017 ACM. ISBN 978-1-4503-4590-3/17/04 . \$ $\$ 15.00$ DOI: http://dx.doi.org/10.1145/3049797.3049818 curse of dimensionality 7 . Recent work in computing stochastic reachable and viable sets aims to circumvent these computational challenges, through approximate dynamic programming 8 10, Gaussian mixtures 9, particle filters 3 10, and convex chance-constrained optimization 35]. These methods have been applied to systems that are at most 6dimensional [8] - far beyond the scope of what is possible with dynamic programming, but are not scalable to larger and more realistic scenarios.

We focus in particular on the forward stochastic reachable set, defined as the smallest closed set that covers all the reachable states. For LTI systems with bounded disturbances, established verification methods $11-13$ can be adapted to overapproximate the forward stochastic reachable set. However, these methods return a trivial result with unbounded disturbances and do not address the forward stochastic reach probability measure, which provides the likelihood of reaching a given set of states.

We present a scalable method to perform forward stochastic reachability analysis of LTI systems with stochastic dynamics, that is, a method to compute the forward stochastic reachable set as well as its probability measure. We show that Fourier transforms can be used to provide exact reachability analysis, for systems with bounded or unbounded disturbances. We provide both iterative and analytical expressions for the probability density, and show that explicit expressions can be derived in some cases.

We are motivated by a particular application: pursuit of a dynamic, non-adversarial target [14. Such a scenario may arise in e.g., the rescue of a lost first responder in a building on fire [15], capture of a non-aggressive UAV in an urban environment [16], or other non-antagonistic situations. Solutions for an adversarial target, based in a two-person, zero-sum differential game, can accommodate bounded disturbances with unknown stochasticity $17-21$, but will be conservative for a non-adversarial target. We seek scalable solutions that synthesize an optimal controller for the nonadversarial scenario, by exploiting the forward reachable set and probability measure for the target. We analyze the convexity properties of the forward stochastic reach probability density and sets, and propose a convex optimization problem to provide the exact probabilistic guarantee of success and the corresponding optimal controller.

The main contributions of this paper are: 1) a method to efficiently compute the forward stochastic reach sets and the corresponding probability measure for linear systems with uncertainty using Fourier transforms, 2) the convexity prop- 
erties of the forward stochastic reach probability measure and sets, and 3) a convex formulation to maximize the probability of capture of a non-adversarial target with stochastic dynamics using the forward stochastic reachability analysis.

The paper is organized as follows: We define the forward stochastic reachability problem and review some properties from probability theory and Fourier analysis in Section 2 Section 3 formulates the forward stochastic reachability analysis for linear systems using Fourier transforms and provides convexity results for the probability measure and the stochastic reachable set. We apply the proposed method to solve the controller synthesis problem in Section 4 and provide conclusions and directions for future work in Section 5

\section{PRELIMINARIES AND PROBLEM FOR- MULATION}

In this section, we review some properties from probability theory and Fourier analysis relevant for our discussion and setup the problems. For detailed discussions on probability theory, see 22 25], and on Fourier analysis, see 26]. We denote random vectors with bold case and non-random vectors with an overline.

\subsection{Preliminaries}

A random vector $\boldsymbol{w} \in \mathbb{R}^{p}$ is defined in a probability space $\left(\mathcal{W}, \sigma(\mathcal{W}), \mathbb{P}_{\boldsymbol{w}}\right)$. Given a sample space $\mathcal{W}$, the sigma-algebra $\sigma(\mathcal{W})$ provides a collection of measurable sets defined over $\mathcal{W}$. The sample space can be either countable (discrete random vector $\boldsymbol{w}$ ) or uncountable (continuous random vector $\boldsymbol{w})$. In this paper, we focus only on absolutely continuous random variables. For an absolutely continuous random vector, the probability measure defines a probability density function $\psi_{\boldsymbol{w}}: \mathbb{R}^{p} \rightarrow \mathbb{R}$ such that given a (Borel) set $\mathcal{B} \in \sigma(\mathcal{W})$, we have $\mathbb{P}_{\boldsymbol{w}}\{\boldsymbol{w} \in \mathcal{B}\}=\int_{\mathcal{B}} \psi_{\boldsymbol{w}}(\bar{z}) d \bar{z}$. Here, $d \bar{z}$ is short for $d z_{1} d z_{2} \ldots d z_{p}$.

We will use the concept of support to define the forward stochastic reach set. The support of a random vector is the smallest closed set that will occur almost surely. Formally, the support of a random vector $\boldsymbol{w}$ is a unique minimal closed set $\operatorname{supp}(\boldsymbol{w}) \in \sigma(\mathcal{W})$ such that 1) $\mathbb{P}_{\boldsymbol{w}}\{\boldsymbol{w} \in \operatorname{supp}(\boldsymbol{w})\}=1$, and 2) if $\mathcal{D} \in \sigma(\mathcal{W})$ such that $\mathbb{P}_{\boldsymbol{w}}\{\boldsymbol{w} \in \mathcal{D}\}=1$, then $\operatorname{supp}(\boldsymbol{w}) \subseteq \mathcal{D}[22$, Section 10, Ex. 12.9]. Alternatively, denoting the Euclidean ball of radius $\delta$ centered at $\bar{z}$ as $\operatorname{Ball}(\bar{z}, \delta)$, we have (1) which is equivalent to 2 via 27 , Proposition 19.3.2],

$$
\begin{aligned}
\operatorname{supp}(\boldsymbol{w}) & =\left\{\bar{z} \in \mathcal{W} \mid \forall \delta>0, \int_{\operatorname{Ball}(\bar{z}, \delta)} \psi_{\boldsymbol{w}}(\bar{z}) d \bar{z}>0\right\} \\
& =\mathcal{W} \backslash\left\{\bar{z} \in \mathcal{W} \mid \exists \delta>0, \psi_{\boldsymbol{w}}(\bar{z})=0 \text { a.e. in } \operatorname{Ball}(\bar{z}, \delta)\right\}
\end{aligned}
$$

For a continuous $\psi_{\boldsymbol{w}},(2)$ is the support of the density 28 , Section 8.8]. Denoting the closure of a set using $\operatorname{cl}(\cdot)$,

$$
\operatorname{supp}(\boldsymbol{w})=\operatorname{support}\left(\psi_{\boldsymbol{w}}\right)=\operatorname{cl}\left(\left\{\bar{z} \in \mathcal{W} \mid \psi_{\boldsymbol{w}}(\bar{z})>0\right\}\right) .
$$

The characteristic function $(\mathrm{CF})$ of a random vector $\boldsymbol{w} \in$ $\mathbb{R}^{p}$ with probability density function $\psi_{\boldsymbol{w}}(\bar{z})$ is

$$
\begin{aligned}
\Psi_{\boldsymbol{w}}(\bar{\alpha}) & \triangleq \mathbb{E}_{\boldsymbol{w}}\left[\exp \left(j \bar{\alpha}^{\top} \boldsymbol{w}\right)\right] \\
& =\int_{\mathbb{R}^{p}} e^{j \bar{\alpha}^{\top} \bar{z}} \psi_{\boldsymbol{w}}(\bar{z}) d \bar{z}=\mathscr{F}\left\{\psi_{\boldsymbol{w}}(\cdot)\right\}(-\bar{\alpha})
\end{aligned}
$$

where $\mathscr{F}\{\cdot\}$ denotes the Fourier transformation operator and $\bar{\alpha} \in \mathbb{R}^{p}$. Given a CF $\Psi_{\boldsymbol{w}}(\bar{\alpha})$, the density function can be computed as

$$
\begin{aligned}
\psi_{\boldsymbol{w}}(\bar{z}) & =\mathscr{F}^{-1}\left\{\Psi_{\boldsymbol{w}}(\cdot)\right\}(-\bar{z}) \\
& =\left(\frac{1}{2 \pi}\right)^{p} \int_{\mathbb{R}^{p}} e^{-j \bar{\alpha}^{\top} \bar{z}} \Psi_{\boldsymbol{w}}(\bar{\alpha}) d \bar{\alpha}
\end{aligned}
$$

where $\mathscr{F}^{-1}\{\cdot\}$ denotes the inverse Fourier transformation operator and $d \bar{\alpha}$ is short for $d \alpha_{1} d \alpha_{2} \ldots d \alpha_{p}$.

We define the $L^{d}\left(\mathbb{R}^{p}\right)$ spaces, $1 \leq d<\infty$, of measurable real-valued functions with finite $L^{d}$ norm. The $L^{d}$ norm of a density $\psi_{\boldsymbol{w}}$ is $\left\|\psi_{\boldsymbol{w}}\right\|_{d} \triangleq\left(\int_{\mathbb{R}^{d}}\left|\psi_{\boldsymbol{w}}(\bar{z})\right|^{d} d \bar{z}\right)^{1 / d}$ where $|\cdot|$ denotes the absolute value. Here, $L^{1}\left(\mathbb{R}^{p}\right)$ is the space of absolutely integrable functions, and $L^{2}\left(\mathbb{R}^{p}\right)$ is the space of square-integrable functions. The Fourier transformation is defined for all functions in $L^{1}\left(\mathbb{R}^{p}\right)$ and all functions in $L^{2}\left(\mathbb{R}^{p}\right)$. Since probability densities are, by definition, in $L^{1}\left(\mathbb{R}^{p}\right)$, CFs exist for every probability density 26 . Section 1]. Let $\boldsymbol{w}_{1}, \boldsymbol{w}_{2} \in \mathbb{R}^{p}$ be random vectors with densities $\psi_{\boldsymbol{w}_{1}}$ and $\psi_{\boldsymbol{w}_{2}}$ and CFs $\Psi_{\boldsymbol{w}_{1}}$ and $\Psi_{\boldsymbol{w}_{2}}$ respectively. By definition, $\psi_{\boldsymbol{w}_{1}}, \psi_{\boldsymbol{w}_{2}} \in L^{1}\left(\mathbb{R}^{p}\right)$. Let $\bar{z}, \bar{z}_{1}, \bar{z}_{2}, \bar{\alpha}, \bar{\alpha}_{1}, \bar{\alpha}_{2} \in \mathbb{R}^{p}, \bar{\beta} \in \mathbb{R}^{n}$.

P1) If $\boldsymbol{x}=\boldsymbol{w}_{1}+\boldsymbol{w}_{2}$, then $\psi_{\boldsymbol{x}}(\bar{z})=\left(\psi_{\boldsymbol{w}_{1}}(\cdot) * \psi_{\boldsymbol{w}_{2}}(\cdot)\right)(\bar{z})$ and $\Psi_{\boldsymbol{x}}(\bar{\alpha})=\Psi_{\boldsymbol{w}_{1}}(\bar{\alpha}) \Psi_{\boldsymbol{w}_{2}}(\bar{\alpha})$ 24. Section 21.11]. Also, $\operatorname{supp}(\boldsymbol{x}) \subseteq \operatorname{cl}\left(\operatorname{supp}\left(\boldsymbol{w}_{1}\right) \oplus \operatorname{supp}\left(\boldsymbol{w}_{2}\right)\right)$ 28, Lemma 8.15]. Here, $*$ denotes convolution and $\oplus$ Minkowski sum.

P2) If $\boldsymbol{x}=F \boldsymbol{w}_{1}+G$ where $F \in \mathbb{R}^{p \times n}, G \in \mathbb{R}^{n}$ are matrices, $\Psi_{\boldsymbol{x}}(\bar{\beta})=\exp \left(j \bar{\beta}^{\top} G\right) \Psi_{\boldsymbol{w}_{1}}\left(F^{\top} \bar{\beta}\right)$ (from 24. Section 22.6] and 26, Equation 1.5]).

P3) If $\boldsymbol{w}_{1}$ and $\boldsymbol{w}_{2}$ are independent vectors, then $\boldsymbol{x}=\left[\begin{array}{ll}\boldsymbol{w}_{1}^{\top} \boldsymbol{w}_{2}^{\top}\end{array}\right]^{\top}$ has probability density $\psi_{\boldsymbol{x}}(\bar{y})=\psi_{\boldsymbol{w}_{1}}\left(\bar{z}_{1}\right) \psi_{\boldsymbol{w}_{2}}\left(\bar{z}_{2}\right), \bar{y}=$ $\left[\begin{array}{ll}\bar{z}_{1}^{\top} & \bar{z}_{2}^{\top}\end{array}\right]^{\top} \in \mathbb{R}^{2 p}$ and CF $\Psi_{\boldsymbol{x}}(\bar{\gamma})=\Psi_{\boldsymbol{w}_{1}}\left(\bar{\alpha}_{1}\right) \Psi_{\boldsymbol{w}_{2}}\left(\bar{\alpha}_{2}\right), \bar{\gamma}=$ $\left[\begin{array}{ll}\bar{\alpha}_{1}^{\top} & \bar{\alpha}_{2}^{\top}\end{array}\right]^{\top} \in \mathbb{R}^{2 p}$ 24. Section 22.4].

P4) The marginal probability density of any group of $k$ components selected from the random vector $\boldsymbol{w}_{1}$ is obtained by setting the remaining $p-k$ Fourier variables in the CF to zero [24, Section 22.4].

An additional assumption of square-integrability of the probability density of the random variable $\boldsymbol{w}_{3} \in \mathbb{R}^{p}$ results in $\psi_{\boldsymbol{w}_{3}} \in L^{1}\left(\mathbb{R}^{p}\right) \cap L^{2}\left(\mathbb{R}^{p}\right)$. Along with Properties P1-P4, $\psi_{\boldsymbol{w}_{3}}$ satisfies the following property:

P5) The Fourier transform preserves the inner product in $L^{2}\left(\mathbb{R}^{p}\right)$ 26. Theorem 2.3]. Given a square-integrable function $\overline{h(\bar{z})}$ with Fourier transform $H(\bar{\alpha})=\mathscr{F}\{h(\cdot)\}(\bar{\alpha})$ and a square-integrable probability density $\psi_{\boldsymbol{w}_{3}}$,

$$
\begin{aligned}
\int_{\mathbb{R}^{p}} \psi_{\boldsymbol{w}_{3}}(\bar{z})^{\dagger} h(\bar{z}) d \bar{z}=\left(\frac{1}{2 \pi}\right)^{p} \int_{\mathbb{R}^{p}}\left(\mathscr{F}\left\{\psi_{\boldsymbol{w}_{3}}(\cdot)\right\}(\bar{\alpha})\right)^{\dagger} \\
\times H(\bar{\alpha}) d \bar{\alpha}
\end{aligned}
$$

Here, $\uparrow$ denotes complex conjugation.

Lemma 1. For square-integrable $\psi_{\boldsymbol{w}_{3}}$ and $h$,

$$
\int_{\mathbb{R}^{p}} \psi_{\boldsymbol{w}_{3}}(\bar{z}) h(\bar{z}) d \bar{z}=\left(\frac{1}{2 \pi}\right)^{p} \int_{\mathbb{R}^{p}} \Psi_{\boldsymbol{w}_{3}}(\bar{\alpha}) H(\bar{\alpha}) d \bar{\alpha} .
$$

Proof: Follows from Property P5, (5), and 24, Section 10.6]. Since probability densities are real functions, $\left(\psi_{\boldsymbol{w}_{3}}(\bar{z})\right)^{\dagger}=\psi_{\boldsymbol{w}_{3}}(\bar{z})$ and $\left(\mathscr{F}\left\{\psi_{\boldsymbol{w}_{3}}(\cdot)\right\}(\bar{\alpha})\right)^{\dagger}=\Psi_{\boldsymbol{w}_{3}}(\bar{\alpha})$. 


\subsection{Problem formulation}

Consider the discrete-time linear time-invariant system,

$$
\boldsymbol{x}[t+1]=A \boldsymbol{x}[t]+B \boldsymbol{w}[t]
$$

with state $\boldsymbol{x}[t] \in \mathcal{X} \subseteq \mathbb{R}^{n}$, disturbance $w[t] \in \mathcal{W} \subseteq \mathbb{R}^{p}$, and matrices $A, B$ of appropriate dimensions. Let $\bar{x}_{0} \in \mathcal{X}$ be the given initial state and $T$ be the finite time horizon. The disturbance set $\mathcal{W}$ is an uncountable set which can be either bounded or unbounded, and the random vector $\boldsymbol{w}[t]$ is defined in a probability space $\left(\mathcal{W}, \sigma(\mathcal{W}), \mathbb{P}_{\boldsymbol{w}}\right)$. The random vector $\boldsymbol{w}[t]$ is assumed to be absolutely continuous with a known density function $\psi_{\boldsymbol{w}}$. The disturbance process $\boldsymbol{w}[\cdot]$ is assumed to be a random process with an independent and identical distribution (IID).

The dynamics in (7) are quite general and includes affine noise perturbed LTI discrete-time systems with known statefeedback based inputs. An additional affine term in (7) can include affine noise perturbed LTI discrete-time systems with known open-loop controllers. For time $\tau \in[1, T]$,

$$
\boldsymbol{x}[\tau]=A^{\tau} \bar{x}_{0}+\mathscr{C}_{n \times(\tau p)} \boldsymbol{W}
$$

with $\mathscr{C}_{n \times(\tau p)}=\left[\begin{array}{lllll}B & A B & A^{2} B & \ldots & A^{\tau-1} B\end{array}\right] \in \mathbb{R}^{n \times(\tau p)}$ and $\boldsymbol{W}=\left[\boldsymbol{w}^{\top}[\tau-1] \boldsymbol{w}^{\top}[\tau-2] \ldots \boldsymbol{w}^{\top}[0]\right]^{\top}$ as a random vector defined by the sequence of random vectors $\{\boldsymbol{w}[t]\}_{t=0}^{t=\tau-1}$. For any given $\tau$, the random vector $\boldsymbol{W}$ is defined in the product space $\left(\mathcal{W}^{\tau}, \sigma\left(\mathcal{W}^{\tau}\right), \mathbb{P}_{W}\right)$ where $\mathcal{W}^{\tau}=X_{t=0}^{\tau} \mathcal{W}$ and $\mathbb{P}_{\boldsymbol{W}}=\prod_{t=0}^{\tau} \mathbb{P}_{\boldsymbol{w}}, \psi_{\boldsymbol{W}}=\prod_{t=0}^{\tau} \psi_{\boldsymbol{w}}$ by the IID assumption of the random process $\boldsymbol{w}[\cdot]$. From (8), the state $\boldsymbol{x}[\cdot]$ is a random process with the random vector at each instant $\boldsymbol{x}[t]$ defined in the probability space $\left(\mathcal{X}, \sigma(\mathcal{X}), \mathbb{P}_{\boldsymbol{x}}^{t, \bar{x}_{0}}\right)$ where the probability measure $\mathbb{P}_{\boldsymbol{x}}^{t, \bar{x}_{0}}$ is induced from $\mathbb{P}_{\boldsymbol{W}}$. We denote the random process originating from $\bar{x}_{0}$ as $\boldsymbol{\xi}\left[\cdot ; \bar{x}_{0}\right]$ where for all $t$, $\boldsymbol{\xi}\left[t ; \bar{x}_{0}\right]=\boldsymbol{x}[t]$, and let $\bar{Z}=\left[\bar{z}^{\top}[\tau-1] \bar{z}^{\top}[\tau-2] \ldots \bar{z}^{\top}[0]\right]^{\top} \in$ $\mathbb{R}^{\tau p}$ denote a realization of the random vector $\boldsymbol{W}$.

An iterative method for the forward stochastic reachability analysis (FSR analysis) is given in 1] 29, Section 10.5]. However, for systems perturbed by continuous random variables, the numerical implementation of the iterative approach becomes erroneous for larger time instants due to the iterative numerical evaluation of improper integrals, motivating the need for an alternative implementable approach.

Problem 1. Given the dynamics (7) with initial state $\bar{x}_{0}$, construct analytical expressions at time instant $\tau$ for

1. the smallest closed set that covers all the reachable states (i.e., the forward stochastic reach set), and

2. the probability measure over the forward stochastic reach set (i.e., the forward stochastic reach probability measure)

that do not require an iterative approach.

We are additionally interested in applying the forward stochastic reachable set (FSR set) and probability measure (FSRPM) to the problem of capturing a non-adversarial target. Specifically, we seek a convex formulation to the problem of capturing a non-adversarial target. This requires convexity of the FSR set and concavity of the objective function defined on the probability of successful capture.

Problem 2. For a finite time horizon, find a) a convex formulation for the maximization of the probability of capture of a non-adversarial target with known stochastic dynamics and initial state, and b) the resulting optimal controller that a deterministic robot must employ when there is a non-zero probability of capture.

Problem 2.a. Characterize the sufficient conditions for logconcavity of the FSRPM and convexity of the FSR set.

\section{FORWARD STOCHASTIC REACHABIL- ITY ANALYSIS}

The existence of forward stochastic reach probability density (FSRPD) for systems of the form (7) has been demonstrated in 29. Section 10.5]. For any $\tau \in[1, T]$, the probability of the state reaching a set $\mathcal{S} \in \sigma(\mathcal{X})$ at time $\tau$ starting at $\bar{x}_{0}$ is defined using the FSRPM $\mathbb{P}_{\boldsymbol{x}}^{\tau, \bar{x}_{0}}$,

$$
\mathbb{P}_{\boldsymbol{x}}^{\tau, \bar{x}_{0}}\{\boldsymbol{x}[\tau] \in \mathcal{S}\}=\int_{\mathcal{S}} \psi_{\boldsymbol{x}}\left(\bar{y} ; \tau, \bar{x}_{0}\right) d \bar{y}, \quad \bar{y} \in \mathbb{R}^{n} .
$$

Since the disturbance set $\mathcal{W}$ is uncountable, we focus on the computation of the FSRPD $\psi_{\boldsymbol{x}}$, and use $\sqrt{9}$ to link it to the FSRPM. We have discussed the countable case in 1 .

We define the forward stochastic reach set (FSR set) as the support of the random vector $\boldsymbol{\xi}\left[\tau ; \bar{x}_{0}\right]=\boldsymbol{x}[\tau]$ at $\tau \in$ $[1, T]$ when the initial condition is $\bar{x}_{0} \in \mathcal{X}$. From $(3)$, for a continuous FSRPD,

$\operatorname{FSReach}\left(\tau, \bar{x}_{0}\right)=\operatorname{cl}\left(\left\{\bar{y} \in \mathcal{X} \mid \psi_{\boldsymbol{x}}\left(\bar{y} ; \tau, \bar{x}_{0}\right)>0\right\}\right) \subseteq \mathcal{X}$.

Lemma 2. $\mathcal{S} \cap \operatorname{FSReach}\left(\tau, \bar{x}_{0}\right)=\emptyset \Rightarrow \mathbb{P}_{\boldsymbol{x}}^{\tau, \bar{x}_{0}}\{\boldsymbol{x}[\tau] \in \mathcal{S}\}=0$.

Proof: Follows from (2).

Note that when the disturbance set $\mathcal{W}$ is unbounded, the definition of the FSR set 10 might trivially become $\mathbb{R}^{n}$. Also, for uncountable $\mathcal{W}$, the probability of the state taking a particular value is zero, and therefore, the superlevel sets of the FSRPD do not have the same interpretation as in the countable case [1. However, given the FSRPD, we can obtain the likelihood that the state of (7) will reach a particular set of interest via (9) and the FSR set via (10).

\subsection{Iterative method for reachability analysis}

We extend the iterative approach for the FSR analysis proposed in [1] for a nonlinear discrete-time systems with discrete random variables to a linear discrete-time system with continuous random variables. This discussion, inspired in part by 29, Section 10.5], helps to develop proofs presented later.

Assume that the system matrix $A$ of (7) is invertible. This assumption holds for continuous-time systems which have been discretized via Euler method. For $\tau \in[0, T-1]$, we have from (7) and Property P1,

$$
\psi_{\boldsymbol{x}}\left(\bar{y} ; \tau+1, \bar{x}_{0}\right)=\left(\psi_{A \boldsymbol{x}}\left(\cdot ; \tau, \bar{x}_{0}\right) * \psi_{B \boldsymbol{w}}(\cdot)\right)(\bar{y})
$$

with

$$
\psi_{A \boldsymbol{x}}\left(\bar{y} ; \tau, \bar{x}_{0}\right)=|A|^{-1} \psi_{\boldsymbol{x}}\left(A^{-1} \bar{y} ; \tau, \bar{x}_{0}\right)
$$

$\psi_{A \boldsymbol{x}}\left(\bar{y} ; \tau, \bar{x}_{0}\right)=(\operatorname{det} A)^{-1} \psi_{\boldsymbol{x}}\left(A^{-1} \bar{y} ; \tau, \bar{x}_{0}\right)$ from 23, Example 8.9] for $\tau \geq 1, \psi_{A x}\left(\bar{y} ; 0, \bar{x}_{0}\right)=\delta\left(\bar{y}-A \bar{x}_{0}\right)$ where $\delta(\cdot)$ is the Dirac-delta function 30. Chapter 5], and $\psi_{B \boldsymbol{w}}$ as the probability density of the random vector $B \boldsymbol{w}$. We use Property P2 and (5) to obtain $\psi_{B \boldsymbol{w}}$ [26. Corollary 1]. Equation (11) is a special case of the result in 29, Section 10.5]. We extend the FSR set computation presented in [1] in the following lemma. 
Lemma 3. For $\tau \in[1, T]$, closed disturbance set $\mathcal{W}$, and the system in (7) with initial condition $\bar{x}_{0}, \operatorname{FSReach}\left(\tau, \bar{x}_{0}\right) \subseteq$ $A\left(\operatorname{FSReach}\left(\tau-1, \bar{x}_{0}\right)\right) \oplus B \mathcal{W}=\left\{A^{\tau} \bar{x}_{0}\right\} \oplus \mathscr{C}_{n \times(\tau p)} \mathcal{W}^{\tau}$.

Proof: Follows from (7), (8), and Property P1. Lemma 3 allows the use of existing reachability analysis schemes designed for bounded non-stochastic disturbance models 11-13 for overapproximating FSR sets. Also, 10 and (11) provide an iterative method for exact FSR analysis.

Note that (11) is an improper integral which must be solved iteratively. For densities whose convolution integrals are difficult to obtain analytically, we would need to rely on numerical integration (quadrature) techniques. Numerical evaluation of multi-dimensional improper integrals is computationally expensive [31, Section 4.8]. Moreover, the quadratures in this method will become increasingly erroneous for larger values of $\tau \in[1, T]$ due to the iterative definition. These disadvantages motivate the need to solve Problem 1 - an approach that provides analytical expressions of the FSRPD, and thereby reduce the number of quadratures required. The iterative method performs well with discrete random vectors as in 1 because discretization for computation can be exact, however, this is clearly not true when the disturbance set is uncountable.

\subsection{Efficient reachability analysis via charac- teristic functions}

We employ Fourier transformation to provide analytical expressions of the FSRPD at any instant $\tau \in[1, T]$. This method involves computing a single integral for the time instant of interest $\tau$ as opposed to the iterative approach in Subsection 3.1 We also show that for certain disturbance distributions like the Gaussian distribution, an explicit expression for the FSRPD can be obtained.

By Property P3 and the IID assumption on the random process $\boldsymbol{w}[\cdot]$, the $\mathrm{CF}$ of the random vector $\boldsymbol{W}$ is

$$
\Psi_{\boldsymbol{W}}(\bar{\alpha})=\prod_{t=0}^{t=\tau-1} \Psi_{\boldsymbol{w}}\left(\bar{\alpha}_{t}\right)
$$

where $\bar{\alpha}=\left[\begin{array}{llll}\bar{\alpha}_{0}^{\top} & \bar{\alpha}_{1}^{\top} \ldots \bar{\alpha}_{\tau-1}^{\top}\end{array}\right]^{\top} \in \mathbb{R}^{(\tau p)}, \bar{\alpha}_{t} \in \mathbb{R}^{p}$ for all $\tau \in$ $[0, \tau-1]$. As seen in (8), the random vector $\boldsymbol{W}$ concatenates the disturbance random process $\boldsymbol{w}[t]$ over $t \in[0, \tau-1]$.

Theorem 1. For any time instant $\tau \in[1, T]$ and an initial state $\bar{x}_{0} \in \mathcal{X}$, the FSRPD $\psi_{\boldsymbol{x}}\left(\cdot ; \tau, \bar{x}_{0}\right)$ of $(7)$ is given by

$$
\begin{aligned}
\Psi_{\boldsymbol{x}}\left(\bar{\alpha} ; \tau, \bar{x}_{0}\right) & =\exp \left(j \bar{\alpha}^{\top}\left(A^{\tau} \bar{x}_{0}\right)\right) \Psi_{\boldsymbol{W}}\left(\mathscr{C}_{n \times(\tau p)}^{\top} \bar{\alpha}\right) \\
\psi_{\boldsymbol{x}}\left(\bar{y} ; \tau, \bar{x}_{0}\right) & =\mathscr{F}^{-1}\left\{\Psi_{\boldsymbol{x}}\left(\bar{\alpha} ; \tau, \bar{x}_{0}\right)\right\}(-\bar{y})
\end{aligned}
$$

where $\bar{y} \in \mathcal{X}, \bar{\alpha} \in \mathbb{R}^{n \times 1}$.

Proof: Follows from Property P2, (5), and (8).

Theorem 1 provides an analytical expression for the FSRPD. Theorem 1 holds even if we relax the identical distribution assumption on the random process $\boldsymbol{w}[t]$ to a timevarying independent disturbance process, provided $\Psi_{\boldsymbol{w}[t]}(\cdot)$ is known for all $t \in[0, \tau-1]$. Using Property P2, Theorem 1 can also be easily extended to include affine noise perturbed LTI discrete-time systems with known open-loop controllers.

Note that the computation of the FSRPD via Theorem 1 does not require gridding of the state space, hence mitigating the curse of dimensionality associated with the traditional gridding-based approaches. When the $\mathrm{CF} \Psi_{\boldsymbol{x}}\left(\bar{\alpha} ; \tau, \bar{x}_{0}\right)$ has the structure of known Fourier transforms, Theorem 1 can be used to provide explicit expressions for the FSRPD (see Proposition 1). In systems where the inverse Fourier transform is not known, the evaluation of (15) can be done via any quadrature techniques that can handle improper integrals. Alternatively, the improper integral can be approximated by the quadrature of an appropriately defined proper integral [31, Chapter 4]. For high-dimensional systems, performance is affected by the scalability of quadrature schemes with dimension. However, Theorem 1 still requires only a single $n$-dimensional quadrature for any time instant of interest $\tau \in[1, T]$. On the other hand, the iterative method proposed in Subsection 3.1 requires $\tau$ quadratures, each $n$ dimensional, resulting in higher computational costs and degradation in accuracy as $\tau$ increases.

One example of a CF with known Fourier transforms arises in Gaussian distributions. We use Theorem 1 to derive an explicit expression for the FSRPD of (7) when perturbed by a Gaussian random vector. Note that the FSRPD in this case can also be computed using the well-known properties on linear combination of Gaussian random vectors 23, Section 9] or the theory of Kalman-Bucy filter 32].

Proposition 1. The system trajectory of (7) with initial condition $\bar{x}_{0}$ and noise process $\boldsymbol{w} \sim \mathcal{N}\left(\bar{\mu}_{\boldsymbol{w}}, \Sigma_{\boldsymbol{w}}\right) \in \mathbb{R}^{p}$ is

$$
\boldsymbol{\xi}\left[\tau ; \bar{x}_{0}\right] \sim \mathcal{N}(\bar{\mu}[\tau], \Sigma[\tau])
$$

where $\tau \in[1, T]$ and

$$
\begin{aligned}
\bar{\mu}[\tau] & =A^{\tau} \bar{x}_{0}+\mathscr{C}_{n \times(\tau p)}\left(\overline{1}_{\tau \times 1} \otimes \bar{\mu}_{\boldsymbol{w}}\right), \\
\Sigma[\tau] & =\mathscr{C}_{n \times(\tau p)}\left(I_{\tau} \otimes \Sigma_{\boldsymbol{w}}\right) \mathscr{C}_{n \times(\tau p)}^{\top} .
\end{aligned}
$$

Proof: For $\bar{\alpha} \in \mathbb{R}^{p}$, the CF of a multivariate Gaussian random vector $\boldsymbol{w}$ is 23. Section 9.3]

$$
\Psi_{\boldsymbol{w}}(\bar{\alpha})=\exp \left(j \bar{\alpha}^{\top} \bar{\mu}_{\boldsymbol{w}}-\frac{\bar{\alpha}^{\top} \Sigma_{\boldsymbol{w}} \bar{\alpha}}{2}\right) .
$$

From the IID assumption of $\boldsymbol{w}[\cdot]$, Property P3, and (19), the $\mathrm{CF}$ of $\boldsymbol{W}$ is

$$
\begin{aligned}
\Psi_{\boldsymbol{W}}(\bar{\alpha}) & =\prod_{t=0}^{t=\tau-1} \exp \left(j \bar{\alpha}_{t}^{\top} \bar{\mu}_{\boldsymbol{w}}-\frac{\bar{\alpha}_{t}^{\top} \Sigma_{\boldsymbol{w}} \bar{\alpha}_{t}}{2}\right) \\
& =\exp \left(j \bar{\alpha}^{\top}\left(\overline{1}_{\tau \times 1} \otimes \bar{\mu}_{\boldsymbol{w}}\right)-\frac{\bar{\alpha}^{\top}\left(I_{\tau} \otimes \Sigma_{\boldsymbol{w}}\right) \bar{\alpha}}{2}\right)
\end{aligned}
$$

where $\bar{\alpha}=\left(\bar{\alpha}_{0}, \bar{\alpha}_{1}, \ldots, \bar{\alpha}_{\tau-1}\right) \in \mathbb{R}^{(\tau p)}$ with $\bar{\alpha}_{t} \in \mathbb{R}^{p}$. Here, $\overline{1}_{p \times q} \in \mathbb{R}^{p \times q}$ is a matrix with all entries as 1 , and $I_{n}$ is the identity matrix of dimension $n$. By $\sqrt{15}$ and $\sqrt{19}, \boldsymbol{W} \sim$ $\mathcal{N}\left(\overline{1}_{\tau \times 1} \otimes \bar{\mu}_{\boldsymbol{w}}, I_{\tau} \otimes \Sigma_{\boldsymbol{w}}\right)$ 26, Corollary 1.22]. From [14), we see that for $\bar{\beta} \in \mathbb{R}^{n}$,

$$
\begin{aligned}
\Psi_{\boldsymbol{x}}\left(\bar{\beta} ; \tau, \bar{x}_{0}\right) & \left.=\exp \left(j \bar{\beta}^{\top}\left(A^{\tau} \bar{x}_{0}\right)\right) \Psi_{\boldsymbol{W}}\left(\mathscr{C}_{n \times(\tau p)}^{\top} \bar{\beta}\right)\right) \\
& =\exp \left(j \bar{\beta}^{\top}\left(A^{\tau} \bar{x}_{0}+\mathscr{C}_{n \times(\tau p)}\left(\overline{1}_{\tau \times 1} \otimes \bar{\mu}_{\boldsymbol{w}}\right)\right)\right) \times \\
\exp & \left(-\frac{\bar{\beta}^{\top} \mathscr{C}_{n \times(\tau p)}\left(I_{\tau} \otimes \Sigma_{\boldsymbol{w}}\right) \mathscr{C}_{n \times(\tau p)}^{\top} \bar{\beta}}{2}\right)
\end{aligned}
$$

Equation 20 is the CF of a multivariate Gaussian random vector 26, Corollary 1.22], and we obtain $\mu_{G}[\tau]$ and $\Sigma_{G}[\tau]$ using 19.

Depending on the system dynamics and time instant of interest $\tau$, we can have $\operatorname{rank}\left(\mathscr{C}_{n \times(\tau p)}\right)<n$. In such cases, the support of the random vector $\boldsymbol{x}[\tau]$ will be restricted to 
sets of lower dimension than $n$ [33. Section 8.5], and certain marginal densities can be Dirac-delta functions. For example, we see that turning off the effect of disturbance in (7) (setting $B=0 \Rightarrow \mathscr{C}_{n \times(\tau p)}=0$ in Theorem 1) yields $\psi_{\boldsymbol{x}}\left(\bar{y} ; \tau, \bar{x}_{0}\right)=\delta\left(\bar{y}-A^{\tau} \bar{x}_{0}\right)$, the trajectory of the corresponding deterministic system. We have used the relation $\mathscr{F}\left\{\delta\left(\bar{y}-\bar{y}_{0}\right)\right\}(\bar{\alpha})=\exp \left(j \bar{\alpha}^{\top} \bar{y}_{0}\right) \quad 30$. Chapters 5,6$]$.

Theorem 1 and $(10)$ provide an analytical expression for the FSRPD and the FSR set respectively, and thereby solve Problem 1 for any density function describing the stochastics of the perturbation $\boldsymbol{w}[t]$ in $(7)$.

\subsection{Convexity results for reachability analysis}

For computational tractability, it is useful to study the convexity properties of the FSRPD and the FSR sets. We define the random vector $\boldsymbol{w}_{B}=B \boldsymbol{w}$ with density $\psi_{\boldsymbol{w}_{B}}$.

Lemma 4. [25, Lemma 2.1] If $\psi_{\boldsymbol{w}}$ is a log-concave distri-

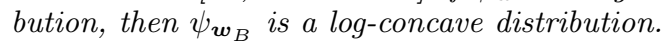

Theorem 2. If $\psi_{\boldsymbol{w}}$ is a log-concave distribution, and $A$ in (7) is invertible, then the FSRPD $\psi_{\boldsymbol{x}}\left(\bar{y} ; \tau, \bar{x}_{0}\right)$ of (7) is logconcave in $\bar{y}$ for every $\tau \in[1, T]$.

Proof: We prove this theorem via induction. First, we need to show that the base case is true, i.e, we need to show that $\psi_{\boldsymbol{x}}\left(\bar{y} ; 1, \bar{x}_{0}\right)$ is log-concave in $\bar{y}$. From (7) and Lemma 4 . we have a log-concave density $\psi_{\boldsymbol{x}}\left(\bar{y} ; 1, \bar{x}_{0}\right)=\psi_{\boldsymbol{w}_{B}}\left(\bar{y}-A \bar{x}_{0}\right)$ since affine transformations preserve log-concavity 34, Section 3.2.4]. Assume for induction, $\psi_{\boldsymbol{x}}\left(\bar{y} ; \tau, \bar{x}_{0}\right)$ is log-concave in $\bar{y}$ for some $\tau \in[1, T]$. We have $\log$-concave $\psi_{A \boldsymbol{x}}\left(\bar{y} ; \tau, \bar{x}_{0}\right)$ from 12. Since convolution preserves log-concavity 34 Section 3.5.2], Lemma 4 and (11) complete the proof.

Corollary 1. If $\psi_{\boldsymbol{w}}$ is a log-concave distribution, FSReach $\left(\tau, \bar{x}_{0}\right)$ of the system (7) is convex for every $\tau \in[1, T], \bar{x}_{0} \in \mathcal{X}$.

Proof: Follows from $[10$ and 25, Theorem 2.5]. Theorem 2 and Corollary 1 solve Problem 2.a.

\section{REACHING A NON-ADVERSARIAL TAR- GET WITH STOCHASTIC DYNAMICS}

In this section, we will leverage the theory developed in this paper to solve Problem 2 efficiently.

We consider the problem of a controlled robot $(\mathrm{R})$ having to capture a stochastically moving non-adversarial target, denoted here by a goal robot $(\mathrm{G})$. The robot $\mathrm{R}$ has controllable linear dynamics while the robot $G$ has uncontrollable linear dynamics, perturbed by an absolutely continuous random vector. The robot $R$ is said to capture robot $G$ if the robot $\mathrm{G}$ is inside a pre-determined set defined around the current position of robot $\mathrm{R}$. We seek an open-loop controller (independent of the current state of robot $\mathrm{G}$ ) for the robot $\mathrm{R}$ which maximizes the probability of capturing robot $\mathrm{G}$ within the time horizon $T$. The information available to solve this problem are the position of the robots $\mathrm{R}$ and $\mathrm{G}$ at $t=0$, the deterministic dynamics of the robot $R$, the perturbed dynamics of the robot $\mathrm{G}$, and the density of the perturbation. We consider a 2-D environment, but our approach can be easily extended to higher dimensions. We perform the FSR analysis in the inertial coordinate frame.

We model the robot $\mathrm{R}$ as a point mass system discretized in time,

$$
\bar{x}_{R}[t+1]=\bar{x}_{R}[t]+B_{R} \bar{u}_{R}[t]
$$

with state (position) $\bar{x}_{R}[t] \in \mathbb{R}^{2}$, input $\bar{u}_{R}[t] \in \mathcal{U} \subseteq \mathbb{R}^{2}$, input matrix $B_{R}=T_{s} I_{2}$ and sampling time $T_{s}$. We define an open-loop control policy $\bar{u}_{R}[t]=\pi_{\text {open, }, \bar{x}_{R}[0]}[t]$ where $\pi_{\text {open, } \bar{x}_{R}[0]}[t]$ depends on the initial condition, that is, $\pi_{\text {open, }, \bar{x}_{R}[0]}:[0, T-1] \rightarrow \mathcal{U}$ is a sequence of control actions for a given initial condition $\bar{x}_{R}[0]$. Let $\mathcal{M}$ denote the set of

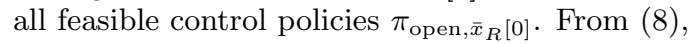

$$
\bar{x}_{R}[\tau+1]=\bar{x}_{R}[0]+\left(\overline{1}_{1 \times \tau} \otimes B_{R}\right) \bar{\pi}_{\tau}, \tau \in[0, T-1]
$$

with the input vector $\bar{\pi}_{\tau}=\left[\bar{u}_{R}^{\top}[\tau-1] \bar{u}_{R}^{\top}[\tau-2] \ldots \bar{u}_{R}^{\top}[0]\right]^{\top}$, $\bar{\pi}_{\tau} \in \overline{\mathcal{M}}_{\tau} \subseteq \mathbb{R}^{(2 \tau)}$, and $\bar{u}_{R}[t]=\pi_{\text {open }, \bar{x}_{R}[0]}[t]$.

We consider two cases for the dynamics of the robot $G$ : 1) point mass dynamics, and 2) double integrator dynamics, both discretized in time and perturbed by an absolutely continuous random vector. In the former case, we presume that the velocity is drawn from a bivariate Gaussian distribution,

$$
\begin{aligned}
\boldsymbol{x}_{G}[t+1] & =\boldsymbol{x}_{G}[t]+B_{\mathrm{G}, \mathrm{PM}} \boldsymbol{v}_{G}[t] \\
\boldsymbol{v}_{G}[t] & \sim \mathcal{N}\left(\bar{\mu}_{G}^{\boldsymbol{v}}, \Sigma_{G}\right) .
\end{aligned}
$$

The state (position) is the random vector $\boldsymbol{x}_{G}[t]$ in the probability space $\left(\mathcal{X}, \sigma(\mathcal{X}), \mathbb{P}_{\boldsymbol{x}_{G}}^{t, \bar{x}_{G}[0]}\right)$ with $\mathcal{X}=\mathbb{R}^{2}$, disturbance matrix $B_{\mathrm{G}, \mathrm{PM}}=B_{R}$, and $\bar{x}_{G}[0]$ as the known initial state of the robot $\mathrm{G}$. The stochastic velocity $\boldsymbol{v}_{G}[t] \in \mathbb{R}^{2}$ has mean vector $\bar{\mu}_{G}^{v}$, covariance matrix $\Sigma_{G}$ and the CF with $\bar{\alpha} \in \mathbb{R}^{2}$ is given in (19). In the latter case, acceleration in each direction is an independent exponential random variable,

$$
\begin{aligned}
\boldsymbol{x}_{G}[t+1] & =A_{\mathrm{G}, \mathrm{DI}} \boldsymbol{x}_{G}[t]+B_{\mathrm{G}, \mathrm{DI}} \boldsymbol{a}[t] \\
(\boldsymbol{a}[t])_{\mathrm{x}} & \sim \operatorname{Exp}\left(\lambda_{\mathrm{ax}}\right), \quad(\boldsymbol{a}[t])_{\mathrm{y}} \sim \operatorname{Exp}\left(\lambda_{\mathrm{ay}}\right) \\
A_{\mathrm{G}, \mathrm{DI}} & =I_{2} \otimes\left[\begin{array}{cc}
1 & T_{s} \\
0 & 1
\end{array}\right], B_{\mathrm{G}, \mathrm{DI}}=I_{2} \otimes\left[\begin{array}{c}
\frac{T_{s}^{2}}{2} \\
T_{s}
\end{array}\right] .
\end{aligned}
$$

The state (position and velocity) is the random vector $\boldsymbol{x}_{G}[t]$ in the probability space $\left(\mathcal{X}_{\mathrm{DI}}, \sigma\left(\mathcal{X}_{\mathrm{DI}}\right), \mathbb{P}_{\boldsymbol{x}_{G}^{t}}^{t, \bar{x}_{G}[0]}\right)$ with $\mathcal{X}_{\mathrm{DI}}=$ $\mathbb{R}^{4}$ and $\bar{x}_{G}[0]$ as the known initial state of the robot $\mathrm{G}$. The stochastic acceleration $\boldsymbol{a}[t]=\left[(\boldsymbol{a}[t])_{\mathrm{x}}(\boldsymbol{a}[t])_{\mathrm{y}}\right]^{\top} \in \mathbb{R}_{+}^{2}=$ $[0, \infty) \times[0, \infty)$ has the following probability density and $\mathrm{CF}$ $\left(\bar{z}=\left[\begin{array}{ll}z_{1} & z_{2}\end{array}\right]^{\top} \in \mathbb{R}_{+}^{2}=[0, \infty) \times[0, \infty), \bar{\alpha}=\left[\begin{array}{ll}\alpha_{1} & \alpha_{2}\end{array}\right]^{\top} \in \mathbb{R}^{2}\right)$,

$$
\begin{aligned}
\psi_{\boldsymbol{a}}(\bar{z}) & =\lambda_{\mathrm{ax}} \lambda_{\mathrm{ay}} \exp \left(-\lambda_{\mathrm{ax}} z_{1}-\lambda_{\mathrm{ay}} z_{2}\right) \\
\Psi_{\boldsymbol{a}}(\bar{\alpha}) & =\frac{\lambda_{\mathrm{ax}} \lambda_{\mathrm{ay}}}{\left(\lambda_{\mathrm{ax}}-j \alpha_{1}\right)\left(\lambda_{\mathrm{ay}}-j \alpha_{2}\right)} .
\end{aligned}
$$

The CF $\Psi_{\boldsymbol{a}}(\bar{\alpha})$ is defined using Property P3 and the CF of the exponential given in 22, Section 26].

Formally, the robot $\mathrm{R}$ captures robot $\mathrm{G}$ at time $\tau$ if $\boldsymbol{x}_{G}[\tau] \in$ $\operatorname{CaptureSet}\left(\bar{x}_{R}[\tau]\right)$. In other words, the capture region of the robot $\mathrm{R}$ is the CaptureSet $(\bar{y}) \subseteq \mathbb{R}^{2}$ when robot $\mathrm{R}$ is at $\bar{y} \in \mathbb{R}^{2}$. The optimization problem to solve Problem 2 is

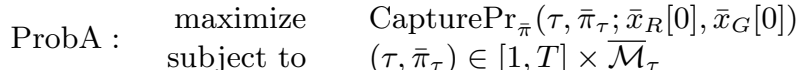

where the decision variables are the time of capture $\tau$ and the control policy $\bar{\pi}$, and the objective function CapturePr $\operatorname{Pr}_{\bar{\pi}}(\cdot)$ gives the probability of robot $\mathrm{R}$ capturing robot $\mathrm{G}$. By (22), an initial state $\bar{x}_{R}[0]$ and the control policy $\bar{\pi}_{t}$ determines a unique $\bar{x}_{R}[\tau]$ for every $\tau$. Using this observation, we define the objective function CapturePr $\operatorname{Pr}_{\bar{\pi}}(\cdot)$ in (27). We obtain $\psi_{\boldsymbol{x}_{G}}$ in 27) using our solution to Problem 1. Theorem 1 . 


$$
\begin{aligned}
& \text { CapturePr } \overline{\bar{\pi}}_{\overline{\bar{x}}}\left(\tau, \bar{\pi}_{\tau} ; \bar{x}_{R}[0], \bar{x}_{G}[0]\right)=\operatorname{CapturePr}_{\bar{x}_{R}}\left(\tau, \bar{x}_{R}[\tau] ; \bar{x}_{G}[0]\right)=\mathbb{P}_{\boldsymbol{x}_{G}^{\tau}}^{\tau, \bar{x}_{G}[0]}\left\{\boldsymbol{x}_{G}[\tau] \in \operatorname{CaptureSet}\left(\bar{x}_{R}[\tau]\right)\right\} \\
& =\int_{\text {CaptureSet }\left(\bar{x}_{R}[\tau]\right)} \psi_{\boldsymbol{x}_{G}}\left(\bar{y} ; \tau, \bar{x}_{G}[0]\right) d \bar{y} .
\end{aligned}
$$

Problem ProbA is equivalent (see [34, Section 4.1.3]) to

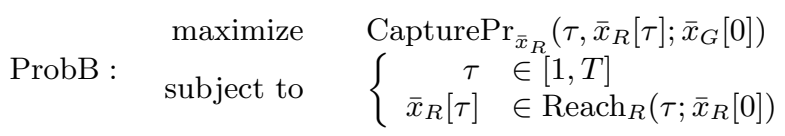

where the decision variables are the time of capture $\tau$ and the position of the robot $\mathrm{R} \bar{x}_{R}[\tau]$ at time $\tau$. From $[22$, we define the reach set for the robot $\mathrm{R}$ at time $\tau$ as

$$
\operatorname{Reach}_{R}\left(\tau ; \bar{x}_{R}[0]\right)=\left\{\bar{y} \in \mathcal{X} \mid \exists \bar{\pi}_{\tau} \in \overline{\mathcal{M}}_{\tau} \text { s.t. } \bar{x}_{R}[\tau]=\bar{y}\right\} .
$$

Several deterministic reachability computation tools are available for the computation of $\operatorname{Reach}_{R}\left(\tau ; \bar{x}_{R}[0]\right)$, like MPT 35 and ET [12]. We will now formulate Problem ProbB as a convex optimization problem based on the results developed in Subsection 3.3

Lemma 5. [11] If the input space $\mathcal{U}$ is convex, the forward reach set $\operatorname{Reach}_{R}\left(\tau ; \bar{x}_{R}[0]\right)$ is convex.

Proposition 2. If $\psi_{\boldsymbol{w}}$ is a log-concave distributions and CaptureSet $(\bar{y})$ is convex for all $\bar{y} \in \mathcal{X}$, then

Capture $\operatorname{Pr}_{\bar{x}_{R}}\left(\tau, \bar{y} ; \bar{x}_{G}[0]\right)$ is log-concave in $\bar{y}$ for all $\tau$.

Proof: From Theorem 2 we know that $\psi_{\boldsymbol{x}}\left(\bar{y} ; \tau, \bar{x}_{R}[0]\right)$ is log-concave in $\bar{y}$ for every $\tau$. The proof follows from 27) since the integration of a log-concave function over a convex set is log-concave 34, Section 3.5.2].

Remark 1. The densities $\psi_{\boldsymbol{v}}$ and $\psi_{\boldsymbol{a}}$ are log-concave since multivariate Gaussian density and exponential distribution (gamma distribution with shape parameter $p=1$ ) are logconcave, and log-concavity is preserved for products [25, Sections 1.4, 2.3] [34, Section 3.5.2].

For any $\tau \in[1, T]$, Proposition 2 and Lemma 5 ensure

$$
\begin{aligned}
\text { Pinimize } & -\log \left(\operatorname{CapturePr}_{\bar{x}_{R}}\left(\tau, \bar{x}_{R}[\tau] ; \bar{x}_{G}[0]\right)\right) \\
\text { subject to } & \bar{x}_{R}[\tau] \in \operatorname{Reach}_{R}\left(\tau ; \bar{x}_{R}[0]\right)
\end{aligned}
$$

is convex with the decision variable $\bar{x}_{R}[\tau]$. Problem ProbC is an equivalent convex optimization problem of the partial maximization with respect to $\bar{x}_{R}[\tau]$ of Problem ProbB since we have transformed the original objective function with a monotone function to yield a convex objective and the constraint sets are identical 34. Section 4.1.3].

We solve Problem ProbB by solving Problem ProbC for each time instant $\tau \in[1, T]$ to obtain $\bar{x}_{R}^{*}[\tau]$ and compute the maximum of the resulting finite set to get $\left(\tau^{*}, \bar{x}_{R}^{*}\left[\tau^{*}\right]\right)$. Since Problem ProbB could be non-convex, this approach ensures a global optimum is found. Note that in order to prevent taking the logarithm of zero, we add an additional constraint to Problem ProbC

$$
\text { CapturePr } \bar{x}_{R}\left(\tau, \cdot ; \bar{x}_{G}[0]\right) \geq \epsilon .
$$

The constraint (28) does not affect its convexity ( $\epsilon$ is a small positive number) from Proposition 2 and the fact that logconcave functions are quasiconcave. Quasiconcave functions have convex superlevel sets 34 , Sections 3.4, 3.5].
Using the optimal solution of Problem ProbB, we can compute the open-loop controller to drive the robot $\mathrm{R}$ from $\bar{x}_{R}[0]$ to $\bar{x}_{R}^{*}\left[\tau^{*}\right]$ by solving Problem ProbD. Defining $\mathscr{C}_{R}=$ $\left(\overline{1}_{1 \times\left(\tau^{*}-1\right)} \otimes B_{R}\right)$ from 22$)$,

$$
\text { ProbD : } \quad \begin{array}{lll}
\text { minimize } & J_{\pi}\left(\bar{\pi}_{\tau^{*}}\right) \\
\bar{\pi}_{\tau^{*}} & \in \overline{\mathcal{M}}_{\tau^{*}} \\
\mathscr{C}_{R} \bar{\pi}_{\tau^{*}} & =\bar{x}_{R}\left[\tau^{*}\right]-\bar{x}_{R}[0]
\end{array}
$$

where the decision variable is $\bar{\pi}_{\tau^{*}}$. The objective function $J_{\pi}\left(\bar{\pi}_{\tau^{*}}\right)=0$ provides a feasible open-loop controller, and $J_{\pi}\left(\bar{\pi}_{\tau^{*}}\right)=\bar{\pi}_{\tau^{*}}^{\top} \bar{R} \bar{\pi}_{\tau^{*}}, \bar{R} \in \mathbb{R}^{\left(2 \tau^{*}\right) \times\left(2 \tau^{*}\right)}$ provides an open-loop controller policy that minimizes the control effort while ensuring that maximum probability of robot $\mathrm{R}$ capturing robot $\mathrm{G}$ is achieved. Solving the optimization problems ProbB and ProbD answers Problem 2.

Our approach to solving Problem 2 is based on our solution to Problem 1 . the Fourier transform based FSR analysis, and Problem 2.a the convexity results of the FSRPD and the FSR sets presented in this paper. In contrast, the iterative approach for the FSR analysis, presented in Subsection 3.1. would yield erroneous Capture $\operatorname{Pr}_{\bar{x}_{R}}\left(\tau, \cdot ; \bar{x}_{G}[0]\right)$ for larger values of $\tau$ due to the heavy reliance on quadrature techniques. Additionally, the traditional approach of dynamic programming based computations [4] would be prohibitively costly for the large FSR sets encountered in this problem due to unbounded disturbances. The numerical implementation of this work is discussed in Subsection 4.3

\subsection{Robot $G$ with point mass dynamics}

We solve Problem ProbB for the system given by (23). Here, the disturbance set is $\mathcal{W}=\mathbb{R}^{2}$.

Lemma 6. For the system given in 23) and initial state of the robot $G$ as $\bar{x}_{G}[0] \in \mathbb{R}^{2}, \operatorname{FSReach}_{G}\left(\tau, \bar{x}_{G}[0]\right)=\mathbb{R}^{2}$ for every $\tau \in[1, T]$.

Proof: Follows from Proposition 1 and 10 .

Proposition 1 provides the FSRPD and Lemma 6 provides the FSR set for the system 23 . The probability of successful capture of the robot $\mathrm{G}$ can be computed using (27) since the FSRPD $\psi_{\boldsymbol{x}_{G}}\left(\cdot ; \tau, \bar{x}_{G}[0]\right)$ is available.

We implement the problem with the following parameters: $T_{s}=0.2, T=20, \bar{\mu}_{G}^{v}=\left[\begin{array}{ll}1.3 & 0.3\end{array}\right]^{\top}, \Sigma_{G}=\left[\begin{array}{cc}0.5 & 0.8 \\ 0.8 & 2\end{array}\right]$, $\bar{x}_{G}[0]=\left[\begin{array}{ll}-3 & 0\end{array}\right]^{\top}, \bar{x}_{R}[0]=\left[\begin{array}{ll}-3 & -2\end{array}\right]^{\top}$ and $\mathcal{U}=[1,2]^{2}$. The capture region of the robot $\mathrm{R}$ is a box centered about the position of the robot $\bar{y}$ with edge length $2 a(a=0.25)$ and edges parallel to the axes - CaptureSet $(\bar{y})=\operatorname{Box}(\bar{y}, a)$, a convex set. We use $J_{\pi}(\bar{\pi})=0$ in Problem ProbD.

Figure 1 shows the evolution of the mean position of the robot $\mathrm{G}$ and the optimal capture position for the robot $\mathrm{R}$ at time instants $4,5,8,14$, and 20 . The contour plots of $\psi_{\boldsymbol{x}_{G}}\left(\cdot ; \tau, \bar{x}_{G}[0]\right)$ are rotated ellipses since $\Sigma_{E}$ is not a diagonal matrix. From (17), the mean position of the robot $\mathrm{G}$ moves in a straight line $\mu_{G}[\tau]$, as it is the trajectory of $23 \mathrm{a}$ when the input is always $\bar{\mu}_{G}^{v}$. The optimal time of capture is $\tau^{*}=$ 5 , the optimal capture position is $\bar{x}_{R}^{*}\left[\tau^{*}\right]=\left[\begin{array}{ll}-1.8 & 0\end{array}\right]^{\top}$, and 


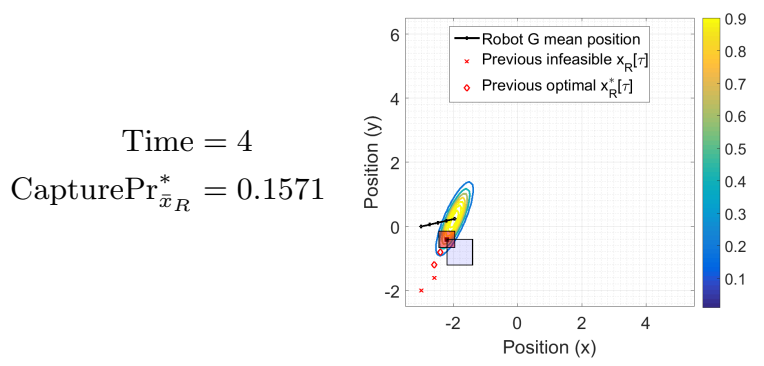

Time $=5$

CapturePr $\operatorname{Pr}_{R}^{*}=0.219$

Time $=6$

CapturePr $\operatorname{s}_{R}^{*}=0.2124$
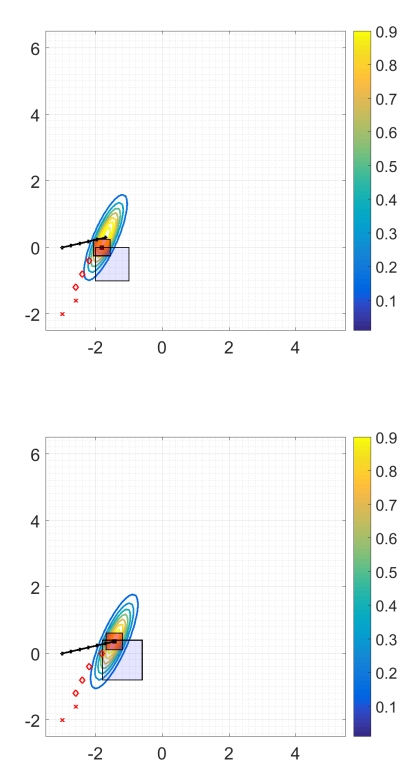

$\begin{aligned} \text { Time } & =14 \\ \text { CapturePr } & =0.1049\end{aligned}$

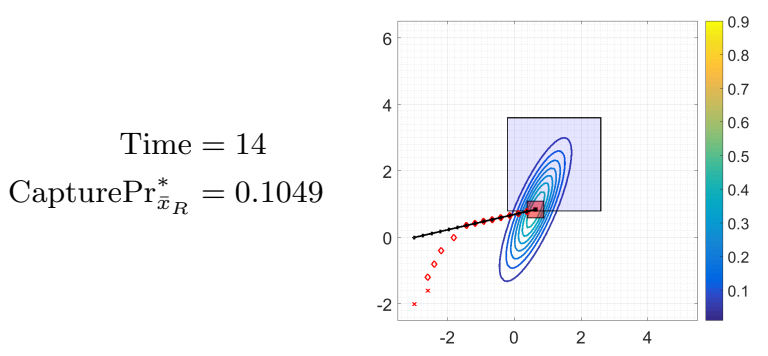

Time $=20$

CapturePr $\operatorname{Pr}_{\bar{x}_{R}}^{*}=0.0624$

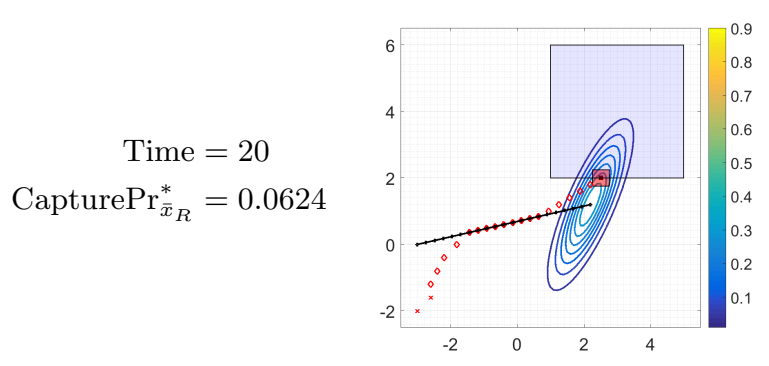

Figure 1: Snapshots of optimal capture positions of the robots $G$ and $R$ when $G$ has point mass dynamics (23). The blue line shows the mean position trajectory of robot $\mathrm{G}$ $\mu_{G}[\tau]$, the contour plot characterizes $\psi_{\boldsymbol{x}_{G}}\left(\cdot ; \tau, \bar{x}_{G}[0]\right)$, the blue box shows the reach set of the robot $\mathrm{R}$ at time $\tau$ $\operatorname{Reach}_{R}\left(\tau, \bar{x}_{R}[0]\right)$, and the red box shows the capture region centered at $\bar{x}_{R}^{*}[\tau]$ CaptureSet $\left(\bar{x}_{R}^{*}[\tau]\right)$.
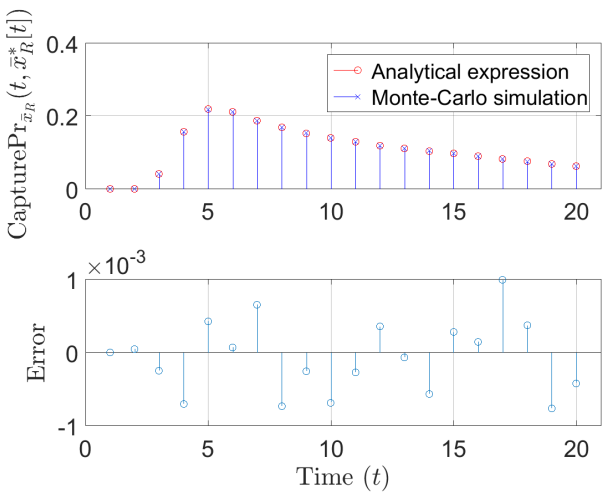

Figure 2: Solution to Problem ProbC for robot G dynamics in 23, and validation of CapturePr $\operatorname{Pr}_{\bar{x}_{R}}\left(\tau, \bar{x}_{R}^{*}[\tau] ; \bar{x}_{G}[0]\right)$ via Monte-Carlo simulations. The optimal capture time is $\tau^{*}=5$ and the likelihood of capture is CapturePr $\operatorname{Pr}_{R}\left(\tau^{*}, \bar{x}_{R}^{*}\left[\tau^{*}\right] ; \bar{x}_{G}[0]\right)=0.219$.

the corresponding probability of robot $\mathrm{R}$ capturing robot $\mathrm{G}$ is 0.219 . Note that at this instant, the reach set of the robot $\mathrm{R}$ does not cover the current mean position of the robot $\mathrm{G}$, $\bar{\mu}\left[\tau^{*}\right]=\left[\begin{array}{ll}-1.7 & 0.3\end{array}\right]^{\top}$ (Figure 1 $)$. While the reach set covers the mean position of robot $\mathrm{G}$ at the next time instant $t=6$, the uncertainty in 23 causes the probability of successful capture to further reduce (Figure 1 1 ). Counterintuitively, attempting to reach the mean $\mu_{G}[\tau]$ is not always best. Figure 2 shows the optimal capture probabilities obtained when solving Problem ProbC for the dynamics 23 .

\subsection{Robot $G$ with double integrator dynamics}

We now consider a more complicated capture problem, in which the disturbance is exponential (hence tracking the mean has little relevance because it is not the mode, the global maxima of the density), and the robot dynamics are more realistic. We solve Problem ProbB for the system given by (24). Here, the disturbance set is $\mathcal{W}=\mathbb{R}_{+}^{2}$. Based on the mean of the stochastic acceleration $\boldsymbol{a}[t]$, the mean position of robot $\mathrm{G}$ has a parabolic trajectory due to the double integrator dynamics, as opposed to the linear trajectory seen in Subsection 4.1 Also, in this case, we do not have an explicit expression for the FSRPD like Proposition 1 Using Theorem 1, we obtain an explicit expression for the CF of the FSRPD. We utilize Lemma 1 to evaluate CapturePr $(\cdot)$.

Analogous to Lemma 6 and Proposition 1, we characterize the FSR set in Lemma 7 and the FSRPD in Proposition 3 We use Lemma 3 to obtain an overapproximation of the FSR set due to the unavailability of FSRPD to use (10).

Lemma 7. For the system given in 24 with initial state $\bar{x}_{G}[0] \in \mathbb{R}^{4}$ of the robot $G$, we have $\mathrm{FSReach}_{G}\left(\tau, \bar{x}_{G}[0]\right) \subseteq$ $\left\{A_{\mathrm{G}, \mathrm{DI}}^{\tau} \bar{x}_{G}[0]\right\} \oplus \mathbb{R}_{+}^{4}$ for every $2 \leq \tau \leq T$, and $\operatorname{FSReach}_{G}\left(1, \bar{x}_{G}[0]\right) \subseteq\left\{A_{\mathrm{G}, \mathrm{DI}} \bar{x}_{G}[0]\right\} \oplus B_{\mathrm{G}, \mathrm{DI}} \mathbb{R}_{+}^{2}$.

Proof: For the dynamics in (24), $\mathscr{C}_{4 \times(2 \tau)}^{\top} \mathbb{R}_{+}^{2 \tau}=\mathbb{R}_{+}^{4}$ since the rank of $\mathscr{C}_{4 \times(2 \tau)}^{\top}$ is 4 for every $\tau \geq 2$, and elements of $\mathscr{C}_{4 \times(2 \tau)}^{\top}$ are nonnegative. For $\tau=1, \mathscr{C}_{4 \times(2 \tau)}^{\top}=B_{\mathrm{G}, \mathrm{DI}}$. Lemma 3 completes the proof. 


$$
\begin{aligned}
\text { Time } & =1 \\
\text { CapturePr } & \operatorname{Pr}_{\bar{x}_{R}}^{*}=0
\end{aligned}
$$

(Infeasible)
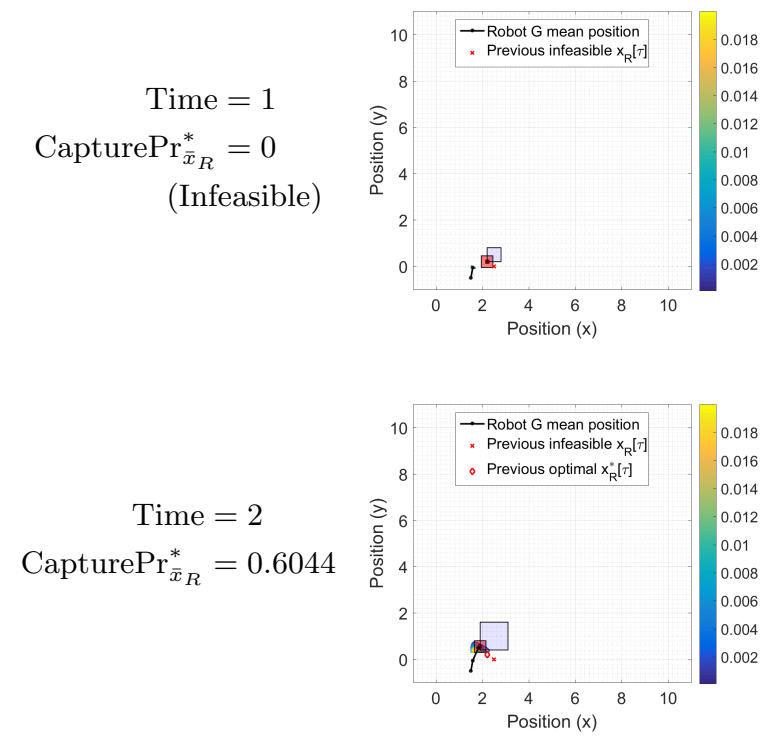

Time $=3$

CapturePr $\dot{x}_{R}^{*}=0.3885$

$\begin{aligned} \text { Time } & =6 \\ \text { CapturePr } & \operatorname{Pr}_{\bar{x}_{R}}^{*}=0.0495\end{aligned}$

Time $=9$

CapturePr r $_{R}^{*}=0.0091$
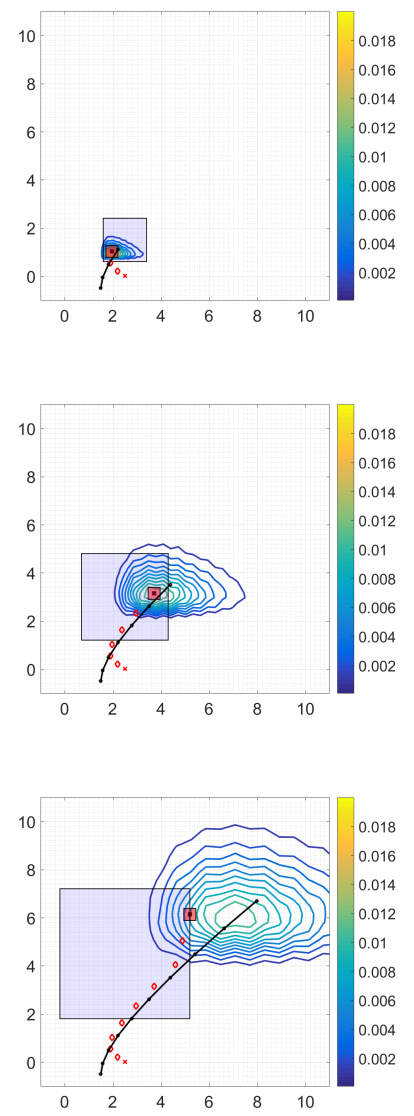

Figure 3: Snapshots of optimal capture positions of the robots $\mathrm{G}$ and $\mathrm{R}$ when $\mathrm{G}$ has double integrator dynamics (24). The blue line shows the mean position trajectory of robot G $\mu_{G}[\tau]$, the contour plot characterizes $\psi_{\boldsymbol{x}_{G}}^{\text {pos }}\left(\cdot ; \tau, \bar{x}_{G}[0]\right)$ via Monte-Carlo simulation, the blue box shows the reach set of the robot $\mathrm{R}$ at time $\tau, \operatorname{Reach}_{R}\left(\tau, \bar{x}_{R}[0]\right)$, and the red box shows the capture region centered at $\bar{x}_{R}^{*}[\tau]$, CaptureSet $\left(\bar{x}_{R}^{*}[\tau]\right)$.
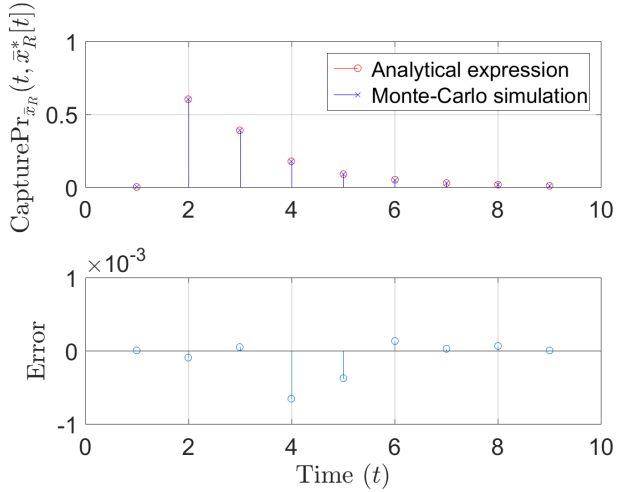

Figure 4: Solution to Problem ProbC for robot G dynamics in 24, and validation of CapturePr $\operatorname{Pr}_{R}\left(\tau, \bar{x}_{R}^{*}[\tau] ; \bar{x}_{G}[0]\right)$ via Monte-Carlo simulations. The optimal capture time is $\tau^{*}=2$ and the capture probability is CapturePr $\operatorname{Pr}_{R}\left(\tau^{*}, \bar{x}_{R}^{*}\left[\tau^{*}\right] ; \bar{x}_{G}[0]\right)=0.6044$.

Proposition 3. The CF of the FSRPD of the robot $G$ for dynamics (24) is

$$
\begin{aligned}
\Psi_{\boldsymbol{x}_{G}}\left(\bar{\beta} ; \tau, \bar{x}_{G}[0]\right) & =\exp \left(j \bar{\beta}^{\top}\left(A_{\mathrm{G}, \mathrm{DI}}^{\tau} \bar{x}_{G}[0]\right)\right) \times \\
& \prod_{t=0}^{\tau-1} \frac{\lambda_{\mathrm{ax}} \lambda_{\mathrm{ay}}}{\left(\lambda_{\mathrm{ax}}-j \bar{\alpha}_{2 t}\right)\left(\lambda_{\mathrm{ay}}-j \bar{\alpha}_{2 t+1}\right)}
\end{aligned}
$$

where $\bar{\alpha}=\mathscr{C}_{4 \times(2 \tau)}^{\top} \bar{\beta} \in \mathbb{R}^{(2 \tau)}$ and $\bar{\beta} \in \mathbb{R}^{4}$. The FSRPD of the robot $G$ is $\psi_{\boldsymbol{x}_{G}}\left(\bar{x} ; \tau, \bar{x}_{G}[0]\right)=\mathscr{F}^{-1}\left\{\Psi_{\boldsymbol{x}_{G}}\left(\cdot ; \tau, \bar{x}_{G}[0]\right)\right\}(-\bar{x})$.

Proof: Apply Theorem 1 to the dynamics (24).

To solve Problem ProbB, we define CapturePr ${ }_{\bar{x}_{R}}(\cdot)$ as in 27). Since we are interested in just the position of robot $\mathrm{G}$, we require only the marginal density of the FSRPD over the position subspace of robot $\mathrm{G}, \psi_{\boldsymbol{x}_{G}}^{\text {pos }}$. By Property P4, we have for $\bar{\gamma}=\left[\begin{array}{ll}\gamma_{1} & \gamma_{2}\end{array}\right] \in \mathbb{R}^{2}$,

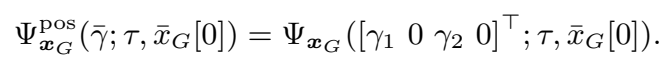

Unlike the case with Gaussian disturbance, explicit expressions for the FSRPD $\psi_{\boldsymbol{x}_{G}}$ or its marginal density $\psi_{\boldsymbol{x}_{G}}^{\text {pos }}$ are unavailable since the Fourier transform 29 is not standard.

Lemma 8. $\psi_{B_{\mathrm{G}, \mathrm{DI}} \boldsymbol{a}}, \psi_{\boldsymbol{x}_{G}} \in L^{1}\left(\mathbb{R}^{4}\right) \cap L^{2}\left(\mathbb{R}^{4}\right)$.

Proof: (For $\left.\psi_{B_{\mathrm{G}, \mathrm{DI}} \boldsymbol{a}}\right)$ By Hölder's inequality 22 , Section 19], $\psi_{\boldsymbol{a}} \in L^{1}\left(\mathbb{R}^{2}\right) \cap L^{2}\left(\mathbb{R}^{2}\right)$. We also have $\psi_{B_{\mathrm{G}, \mathrm{DI}} \boldsymbol{a}}\left(z_{1}, z_{2}, z_{3}, z_{4}\right)=\delta\left(z_{3}-\frac{\dot{T}_{s} z_{4}}{2}\right) \delta\left(z_{1}-\frac{T_{s} z_{2}}{2}\right) \psi_{\boldsymbol{z}_{24}}\left(z_{2}, z_{4}\right)$ where $\boldsymbol{z}_{24}=\left[\begin{array}{ll}z_{2} & z_{4}\end{array}\right]^{\top}=T_{s} \boldsymbol{a} \in \mathbb{R}^{2}$ and $\psi_{\boldsymbol{z}_{24}}\left(z_{2}, z_{4}\right)=$ $T_{s}^{-2} \psi_{\boldsymbol{a}}\left(\frac{z_{2}}{T_{s}}, \frac{z_{4}}{T_{s}}\right)$ from 12$\}$. For $i=\{1,2\},\left\|\psi_{B_{\mathrm{G}, \mathrm{DI}} \boldsymbol{a}}\right\|_{i}=$ $\left\|\psi_{\boldsymbol{z}_{24}}\right\|_{i}=T_{s}^{2-2 i}\left\|\psi_{\boldsymbol{a}}\right\|_{i}<\infty$ completing the proof.

(For $\psi_{\boldsymbol{x}_{G}}$ ) Via induction using (11) (similar to the proof of Theorem 21. Note that functions in $L^{1}\left(\mathbb{R}^{4}\right) \cap L^{2}\left(\mathbb{R}^{4}\right)$ are closed under convolution [26, Theorem 1.3].

Lemma 9. $\psi_{\boldsymbol{x}_{G}}^{\text {pos }}\left(\bar{x} ; \tau, \bar{x}_{G}[0]\right) \in L^{1}\left(\mathbb{R}^{2}\right) \cap L^{2}\left(\mathbb{R}^{2}\right)$.

Proof: For $i=\{1,2\}$, we have from $30,\left\|\psi_{\boldsymbol{x}_{G}}^{\text {pos }}\right\|_{i}=$ $\left\|\psi_{\boldsymbol{x}_{G}}\right\|_{i}$, and from Lemma $8\left\|\psi_{\boldsymbol{x}_{G}}\right\|_{i}<\infty$.

Similar to Subsection 4.1 we define a convex capture region CaptureSet $\left(\bar{y}_{R}\right)=\operatorname{Box}\left(\bar{y}_{R}, a\right) \subseteq \mathbb{R}^{2}$ where $\bar{y}_{R} \in$ $\mathbb{R}^{2}$ is the state of the robot $\mathrm{R}$. We define $h\left(\bar{y} ; \bar{y}_{R}, a\right)=$ $\mathbf{1}_{\mathrm{Box}\left(\bar{y}_{R}, a\right)}(\bar{y})$ as the indicator function corresponding to a 2$\mathrm{D}$ box centered at $\bar{y}_{R}$ with edge length $2 a>0$ with $h(\bar{y})=1$ 
if $\bar{y} \in$ CaptureSet $\left(\bar{y}_{R}\right)$ and zero otherwise. The Fourier transform of $h$ is a product of sinc functions shifted by $\bar{y}_{R}$ (follows from Property P2 and [30, Chapter 13])

$$
\begin{aligned}
H\left(\bar{\gamma} ; \bar{y}_{R}, a\right) & =\mathscr{F}\left\{h\left(\cdot ; \bar{y}_{R}, a\right)\right\}(\bar{\gamma}) \\
& =4 a^{2} \exp \left(-j \bar{y}_{R}^{\top} \bar{\gamma}\right) \frac{\sin \left(a \gamma_{1}\right) \sin \left(a \gamma_{2}\right)}{\gamma_{1} \gamma_{2}} .
\end{aligned}
$$

Clearly, $h$ is square-integrable, and from Lemmas 1 and 9 , we define CapturePr $\bar{x}_{\bar{x}_{R}}(\cdot)$ in (33). Equation (33) is evaluated using (29), (30), and (31). We use (33) as opposed (32) due to the unavailability of an explicit expression for $\psi_{\boldsymbol{x}_{G}}^{\mathrm{pos}}$. The numerical evaluation of the inverse Fourier transform of $\Psi_{\boldsymbol{x}_{G}}^{\text {pos }}$ to compute 32 will require two quadratures, resulting in a higher approximation error as compared to (33).

We implement the problem with the following parameters: $T_{s}=0.2, T=9, a=0.25, \lambda_{\text {ax }}=0.25, \lambda_{\text {ay }}=0.45, \bar{x}_{G}[0]=$ $\left[\begin{array}{lll}1.5 & 0 & -0.5\end{array}\right]^{\top}, \bar{x}_{R}[0]=\left[\begin{array}{ll}2.5 & 0\end{array}\right]^{\top}$, and $\mathcal{U}=[-1.5,1.5] \times$ $[1,4]$. We use $J_{\pi}(\bar{\pi})=0$ in Problem ProbD.

Figure 3 shows the evolution of the mean position of the robot $\mathrm{G}$ and the optimal capture position for the robot $\mathrm{R}$ at time instants $1,2,3,6$, and 9 . For every $\tau \in[1, T]$, the contour plots of $\psi_{\boldsymbol{x}_{G}}^{\text {pos }}\left(\cdot ; \tau, \bar{x}_{G}[0]\right)$ were estimated via MonteCarlo simulation since evaluating $\psi_{\boldsymbol{x}_{G}}^{\text {pos }}\left(\cdot ; \tau, \bar{x}_{G}[0]\right)$ via over a grid is computationally expensive. Note that the mean position of the robot $\mathrm{G}$ does not coincide with the mode of $\psi_{\boldsymbol{x}_{G}}^{\text {pos }}\left(\cdot ; \tau, \bar{x}_{G}[0]\right)$ in contrast to the problem discussed in Subsection 4.1 The optimal time of capture is at $\tau^{*}=2$, the optimal capture position is $\bar{x}_{R}^{*}\left[\tau^{*}\right]=\left[\begin{array}{ll}1.9 & 0.55\end{array}\right]^{\top}$, and the corresponding probability of robot $\mathrm{R}$ capturing robot $\mathrm{G}$ is 0.6044 (Figure 3b). Figure 4 shows the optimal capture probabilities obtained when solving Problem ProbC for the dynamics 24, and the validation of the results.

\subsection{Numerical implementation and analysis}

All computations in this paper were performed using MAT$\mathrm{LAB}$ on an Intel Core i7 $\mathrm{CPU}$ with $3.4 \mathrm{GHz}$ clock rate and 16 GB RAM. The MATLAB code for this work is available at http://hscl.unm.edu/files/code/HSCC17.zip

We solved Problem ProbC using MATLAB's built-in functions - fmincon for the optimization, mvncdf to compute the objective (27) for the case in Subsection 4.1, integral to compute the objective (33) for the case in Subsection 4.2 . and max to compute the global optimum of Problem ProbB. In both the sections, we used MPT for the reachable set calculation and solved Problem ProbD using CVX 36. Using Lemma 2, the FSR sets restrict the search while solving Problem ProbC. All geometric computations were done in the facet representation. We computed the initial guess for the optimization of Problem ProbC by performing Euclidean projection of the mean to the feasible set using CVX 34 , Section 8.1.1]. Since computing the objective was costly, this operation saved significant computational time. The MonteCarlo simulation used 500, 000 particles. No offline computations were done in either of the cases.

The overall computation of Problem ProbB and ProbD for the case in Subsection 4.1 took 5.32 seconds for $T=20$. Since Proposition 1 provides explicit expressions for the FSRPD, the evaluation of the FSRPD for any given point $\bar{y} \in \mathcal{X}$ takes 1.6 millseconds on average. For the case in Subsection 4.2 the overall computation took 488.55 seconds ( $\sim 8$ minutes) for $T=9$. The numerical evaluation of the improper integral (33) is the major cause of increase in runtime. The evaluation of the FSRPD for any given point $\bar{y} \in \mathcal{X}$ using $(5)$ takes about 10.5 seconds, and the runtime and the accuracy depend heavily on the point $\bar{y}$ as well as the bounds used for the integral approximation. However, the evaluation of Capture $\operatorname{Pr}_{\bar{x}_{R}}(\cdot)$ using (33) is much faster (0.81 seconds) because $H\left(\bar{\gamma} ; \bar{y}_{R}, a\right)$ is a decaying, 2-D sinc function (decaying much faster than the $\mathrm{CF}$ ).

The decaying properties of the integrand in $(33)$ and CFs in general permits approximating the improper integrals in (5) and (33) by as a proper integral with suitably defined finite bounds. The tradeoff between accuracy and computational speed, common in quadrature techniques, dictates the choice of the bound. A detailed analysis of various quadrature techniques, their computational complexity, and their error analysis can be found in 31. Chapter 4].

\section{CONCLUSIONS AND FUTURE WORK}

This paper provides a method for forward stochastic reachability analysis using Fourier transforms. The method is applicable to uncontrolled stochastic linear systems. Fourier transforms simplify the computation and mitigate the curse of dimensionality associated with gridding the state space. We also analyze several convexity results associated with the FSRPD and FSR sets. We demonstrate our method on the problem of controller synthesis for a controlled robot pursuing a stochastically moving non-adversarial target.

Future work includes exploration of various quadrature techniques like particle filters for high-dimensional quadratures, and extension to a model predictive control framework and to discrete random vectors (countable disturbance sets). Multiple pursuer applications will also be investigated.

\section{ACKNOWLEDGEMENTS}

The authors thank Prof. M. Hayat for discussions on Fourier transforms in probability theory and the reviewers for their insightful comments.

This material is based upon work supported by the National Science Foundation, under Grant Numbers CMMI1254990, CNS-1329878, and IIS-1528047. Any opinions, findings, and conclusions or recommendations expressed in this material are those of the authors and do not necessarily reflect the views of the National Science Foundation.

\section{REFERENCES}

[1] Baisravan HomChaudhuri, Abraham P. Vinod, and Meeko M. K. Oishi. Computation of forward stochastic reach sets: Application to stochastic, dynamic obstacle avoidance. In Proc. American Control Conf., 2017. (accepted).

[2] Nick Malone, Kendra Lesser, Meeko Oishi, and Lydia Tapia. Stochastic reachability based motion planning for multiple moving obstacle avoidance. In Proc. Hybrid Syst.: Comput. and Control, pages 51-60, 2014.

[3] Kendra Lesser, Meeko Oishi, and R. Scott Erwin. Stochastic reachability for control of spacecraft relative motion. In Proc. IEEE Conf. on Decision and Control, pages 4705-4712, 2013.

[4] Sean Summers and John Lygeros. Verification of discrete time stochastic hybrid systems: A stochastic reach-avoid decision problem. Automatica, 46(12):1951-1961, 2010.

[5] Nikolaos Kariotoglou, Davide M Raimondo, Sean Summers, and John Lygeros. A stochastic reachability 


$$
\begin{aligned}
\text { CapturePr } \bar{x}_{R}\left(\tau, \bar{x}_{R}[\tau] ; \bar{x}_{G}[0]\right) & =\int_{\mathbb{R}^{2}} \psi_{\boldsymbol{x}_{G}}^{\text {pos }}\left(\bar{x} ; \tau, \bar{x}_{G}[0]\right) h\left(\bar{x} ; \bar{x}_{R}[\tau], a\right) d \bar{x} \\
& =\left(\frac{1}{2 \pi}\right)^{2} \int_{\mathbb{R}^{2}} \Psi_{\boldsymbol{x}_{G}}^{\text {pos }}\left(\bar{\gamma} ; \tau, \bar{x}_{G}[0]\right) H\left(\bar{\gamma} ; \bar{x}_{R}[\tau], a\right) d \bar{\gamma}
\end{aligned}
$$

framework for autonomous surveillance with pan-tilt-zoom cameras. In European Control Conf., pages 1411-1416, 2011.

[6] Alessandro Abate, Maria Prandini, John Lygeros, and Shankar Sastry. Probabilistic reachability and safety for controlled discrete time stochastic hybrid systems. Automatica, 44(11):2724-2734, 2008.

[7] Alessandro Abate, Saurabh Amin, Maria Prandini, John Lygeros, and Shankar Sastry. Computational approaches to reachability analysis of stochastic hybrid systems. In Proc. Hybrid Syst.: Comput. and Control, pages 4-17, 2007.

[8] Nikolaos Kariotoglou, Sean Summers, Tyler Summers, Maryam Kamgarpour, and John Lygeros. Approximate dynamic programming for stochastic reachability. In European Control Conf., pages 584-589, 2013.

[9] Nikolaos Kariotoglou, Kostas Margellos, and John Lygeros. On the computational complexity and generalization properties of multi-stage and stage-wise coupled scenario programs. Syst. and Control Lett., 94:63-69, 2016.

[10] Giorgio Manganini, Matteo Pirotta, Marcello Restelli, Luigi Piroddi, and Maria Prandini. Policy search for the optimal control of Markov Decision Processes: A novel particle-based iterative scheme. IEEE Trans. Cybern., pages 1-13, 2015.

[11] Michal Kvasnica, Bálint Takács, Juraj Holaza, and Deepak Ingole. Reachability analysis and control synthesis for uncertain linear systems in MPT. IFAC Symp. on Robust Control D., 48(14):302-307, 2015.

[12] Alex A. Kurzhanskiy and Pravin Varaiya. Ellipsoidal toolbox. Technical Report UCB/EECS-2006-46, EECS Department, University of California, Berkeley, 2006.

[13] Antoine Girard. Reachability of uncertain linear systems using zonotopes. In Proc. Hybrid Syst.: Comput. and Control, pages 291-305, 2005.

[14] Geoffrey Hollinger, Sanjiv Singh, Joseph Djugash, and Athanasios Kehagias. Efficient multi-robot search for a moving target. Int'l J. Robotics and Research, 28(2):201-219, 2009.

[15] Vijay Kumar, Daniela Rus, and Sanjiv Singh. Robot and sensor networks for first responders. IEEE Pervasive computing, 3(4):24-33, 2004.

[16] Christopher Geyer. Active target search from UAVs in urban environments. In Proc. IEEE Int'l Conf. Robotics and Autom., pages 2366-2371, 2008.

[17] Ian Mitchell and Claire J. Tomlin. Level set methods for computation in hybrid systems. In Proc. Hybrid Syst.: Comput. and Control, pages 310-323, 2000.

[18] Claire J. Tomlin, John Lygeros, and Shankar Sastry. A game theoretic approach to controller design for hybrid systems. Proc. IEEE, 88(7):949-970, 2000.

[19] Claire J. Tomlin, Ian Mitchell, Alexandre M. Bayen, and Meeko Oishi. Computational techniques for the verification of hybrid systems. Proc. IEEE, 91(7):986-1001, 2003.

[20] Olivier Bokanowski, Nicolas Forcadel, and Hasnaa Zidani. Reachability and Minimal Times for State Constrained Nonlinear Problems without Any Controllability Assumption. SIAM J. of Control and Optimization, 48(7):4292-4316, 2010.

[21] Haomiao Huang, Jerry Ding, Wei Zhang, and Claire J. Tomlin. Automation-assisted capture-the-flag: A differential game approach. IEEE Trans. Control Syst. Technol., 23:1014-1028, 2015.

[22] Patrick Billingsley. Probability and measure. Wiley, New York, 3 edition, 1995.

[23] John A Gubner. Probability and random processes for electrical and computer engineers. Cambridge University Press, New York; Cambridge, 2006.

[24] Harald Cramér. Mathematical methods of statistics (PMS-9). Princeton university press, 9 edition, 1961.

[25] Sudhakar Dharmadhikari and Kumar Joag-Dev. Unimodality, convexity, and applications. Elsevier, 1988.

[26] Elias M Stein and Guido L Weiss. Introduction to Fourier analysis on Euclidean spaces, volume 1. Princeton University Press, 1971.

[27] Terence Tao. Analysis II. Hindustan Book Agency, 2 edition, 2009.

[28] Jean-Paul Penot. Analysis: From Concepts to Applications. Springer, 1 edition, 2016.

[29] Andrzej Lasota and Michael C Mackey. Chaos, fractals, and noise: stochastic aspects of dynamics, volume 97. Springer Science \& Business Media, 2013.

[30] Ron Bracewell. The Fourier transform and its applications. McGraw-Hill, Inc., 1986.

[31] William H. Press, Saul A. Teukolsky, William T. Vetterling, and Brian P. Flannery. Numerical recipes: The art of scientific computing. Cambridge University Press, New York, NY, USA, 3 edition, 2007.

[32] Peter Dorato, Vito Cerone, and Chaouki Abdallah. Linear-quadratic control: An introduction. Simon \& Schuster, 1994.

[33] Y.S. Chow and H. Teicher. Probability Theory: Independence, Interchangeability, Martingales. Springer Texts in Statistics. Springer New York, 1997.

[34] Stephen P. Boyd and Lieven Vandenberghe. Convex optimization. Cambridge University Press, Cambridge, UK ; New York, 2004.

[35] Martin Herceg, Michal Kvasnica, Colin N. Jones, and Manfred Morari. Multi-Parametric Toolbox 3.0. In European Control Conf., pages 502-510, 2013. http://control.ee.ethz.ch/ ${ }^{\sim m p t}$

[36] Michael Grant and Stephen Boyd. CVX: MATLAB software for disciplined convex programming, version 2.1. http://cvxr.com/cvx, 2014. 